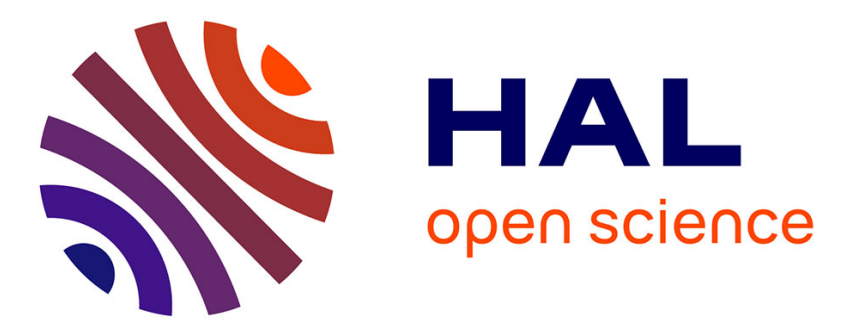

\title{
La religion à toutes fins parlementaires pratiques : comment l'islam est thématisé à l'Assemblée du Peuple syrienne
}

Souhaïl Belhadj, Baudouin Dupret

\section{To cite this version:}

Souhail Belhadj, Baudouin Dupret. La religion à toutes fins parlementaires pratiques: comment l'islam est thématisé à l'Assemblée du Peuple syrienne. Maghreb-Machrek, 2008, 4 (198), pp.31-41. 10.3917/machr.198.0031 . halshs-00375030

\section{HAL Id: halshs-00375030 \\ https://shs.hal.science/halshs-00375030}

Submitted on 19 Apr 2009

HAL is a multi-disciplinary open access archive for the deposit and dissemination of scientific research documents, whether they are published or not. The documents may come from teaching and research institutions in France or abroad, or from public or private research centers.
L'archive ouverte pluridisciplinaire HAL, est destinée au dépôt et à la diffusion de documents scientifiques de niveau recherche, publiés ou non, émanant des établissements d'enseignement et de recherche français ou étrangers, des laboratoires publics ou privés.

\section{(ㅇ)(1) $\$$}

Distributed under a Creative Commons Attribution - NonCommercial - NoDerivatives 44.0 


\section{LA RELIGION À TOUTES FINS PARLEMENTAIRES PRATIQUES: \\ COMMENT L'ISLAM EST THÉMATISÉ À l'Assemblée du Peuple syrienne}

SOuhaïl BELHADJ et Baudouin DUPRET *

Nous voudrions décrire comment une question touchant de près à l'islam et à la charia, à savoir un amendement de la loi du statut personnel sur la question du droit de garde des enfants, est investie par des parlementaires syriens. Ceux-ci s'orientent vers deux types de contraintes : thématique et procédurale. Nous tendons donc à montrer comment le traitement d'une question marquée religieusement est sensible au contexte (institutionnel) de son évocation. L'exercice de l'activité législative par un conseil représentatif remonte, en Syrie, à 1919. Sa désignation, son mode de constitution et l'étendue de ses prérogatives ont fluctué tout au long d'une histoire politique mouvementée.

La Constitution de la République arabe syrienne de 1973 prévoit que le pouvoir législatif est dévolu à l'Assemblée du Peuple (Majlis al-cha'b). La chambre compte 250 sièges de députés élus pour quatre ans. Plus de la moitié de ces sièges (167) sont réservés au parti Ba'th et au Front national progressiste (FNP), une coalition de huit partis qui lui sont affiliés. L'une des principales fonctions de l'Assemblée est d'approuver, certains diront d'enregistrer, le budget, les lois, les accords et traités internationaux engageant la souveraineté nationale et la population. La Constitution confère également à l'Assemblée du Peuple le pouvoir d'interpeller le gouvernement ou un

"Souhaïl Belhadj (IEP Paris), Baudouin Dupret (CNRS, ISP Cachan). 
ministre par le vote d'une motion de censure. Si cette dernière est adoptée, le cabinet ou le ministre en question doit présenter sa démission, bien que le gouvernement soit responsable devant le président de la République.

Le Parlement est la seule institution syrienne dont les membres soient élus à la faveur d'un scrutin national (à majorité simple à un tour). C'est aussi la seule assemblée élue qui joue un rôle dans le processus de désignation du chef de l'État. Dans la symbolique du pouvoir syrien, les masses sont à la source de la légitimité et elles doivent donner périodiquement leur aval à la mise en œuvre de la politique générale du pays. La loi électorale fixe les modalités générales du scrutin législatif, mais c'est le commandement régional du Ba'th, en accord avec la présidence de la République, qui décide de la proportion d' " indépendants ». Cette catégorie désigne les candidats autorisés à se faire élire sans appartenir au FNP. Réduite à l'extrême dans les années 1980, cette proportion atteint au moins $30 \%$ à la faveur de la très relative libéralisation politique entamée en 1990. Ainsi, l'Assemblée qui discute le projet de loi dont cet article traite et qui est élue en 2003, en comptait 83. Ces derniers, certes adoubés par le régime, représentent des groupes d'intérêts susceptibles d'obtenir l'amendement de textes dans un sens non prévu par les caciques du Parti. En outre, les parlementaires FNP n'obéissent pas toujours à la discipline du Parti et s'affranchissent parfois des dogmes idéologiques.

La capacité de l'Assemblée à influer sur les « grandes options de la politique syrienne » est réduite, en tout cas dans les domaines de la réforme de l'État, à des questions stratégiques et de la transformation de la structure économique. Ce régime est donc un régime présidentiel par excellence et le pouvoir exécutif connaît peu d'obstacles à son surcroît d'influence. Malgré tout, la Constitution fait de l'Assemblée du peuple la seule institution limitative - formellement, potentiellement, mais aussi de fait - de l'extension du pouvoir présidentiel. Elle représente également une contrainte de type organique à l'orientation et au champ d'application de la politique en général, mais surtout des politiques publiques. Elle est, de facto, le pendant du gouvernement syrien en ce qui concerne l'initiative des lois, surtout celles touchant aux questions de morale et de société.

Le projet de loi à l'étude dans cet article est un exemple de l'inscription de l'Assemblée du Peuple dans le complexe institutionnel et le jeu politique. À l'instigation de ce projet, on trouve le gouvernement et, plus précisément, le ministre de la Justice, M. Nizar al-'Asasi, lui-même ancien député. Le Premier ministre lui a associé Ghada al-Jabi, ministre du Travail et des Affaires sociales. Le cabinet souhaitait que Mme al-Jabi soutienne ce projet en raison du crédit dont elle bénéficiait auprès de la classe politique du pays et, particulièrement, auprès des députés, en tant qu'ancienne présidente de l'Union générale des femmes syriennes. Le projet propose de modifier l'âge de la garde des enfants par leur mère en cas de divorce. Il s'agit donc d'un amendement à ce que l'on appelle le droit du statut personnel, à savoir le 
droit régissant la famille et, plus particulièrement, le mariage, le divorce, la filiation et l'héritage.

La Syrie a adopté son Code de statut personnel le 7 septembre 1953 (loi $\mathrm{n}^{\circ} 59$ de l'année 1953). Ce texte a été amendé par la loi no 34 de l'année 1975. Parallèlement à la codification du droit syrien, le pouvoir judiciaire a été organisé et unifié en un système unique de cours et tribunaux. Les tribunaux de la charia, intégrés au nouveau système, n'ont pas cessé pour autant de conserver une existence distincte. Le statut personnel des Syriens est fonction de leur confession religieuse individuelle. Le décret-loi $\mathrm{n}^{\circ} 60 \mathrm{de}$ l'année 1936, tel qu'amendé, définit les confessions reconnues en Syrie. Bien qu'il soit rédigé dans un langage abstrait et universel, le Code présuppose l'existence de lois autonomes pour chacune des 17 confessions reconnues. Autrement dit, il suppose que toutes les confessions suivent un seul et même code, alors qu'en fait chacune suit sa propre loi. Le principe veut, en effet, que le Code de statut personnel s'applique à tous les Syriens (art. 306), sauf dans les cas stipulés aux articles 307 et 308. L'article 307 vise la communauté druze et l'article 308, les communautés chrétiennes et juives. En somme, le Code de statut personnel est supposé être le droit commun de tous les Syriens, mais ses nombreuses exceptions limitent le champ d'application de ses dispositions aux musulmans.

L'amendement de 1975 du Code de statut personnel a introduit des changements importants sur les questions de polygamie, de dot, de pension d'allaitement, d'élévation de l'âge de la garde des enfants par leur mère, d'entretien des enfants et de divorce par consentement $\left(\mathrm{khul} \mathrm{l}^{\prime}\right.$. S'agissant de l'âge de la garde des enfants, l'article 146 du Code de statut personnel, tel qu'amendé par l'article 19 de la loi n 34 de l'année 1975, stipule que « la durée de la garde [par la mère] s'achève quand le garçon a atteint l'âge de neuf ans et la fille [celui] de onze [ans] ». Précédemment, l'âge était fixé à sept ans pour le garçon et neuf ans pour la fille. En 2003, la même disposition fut à nouveau amendée par la loi n ${ }^{\circ} 18$, votée par le Parlement le 19 octobre et promulguée par le président le 25 octobre. Larticle 146 du Code de statut personnel est désormais formulé de la manière suivante: "la durée de la garde [par la mère] s'achève quand le garçon a atteint l'âge de treize ans et la fille [celui] de quinze [ans] ». C'est à ce débat que nous allons à présent prêter attention.

En nous appuyant sur des extraits de ce débat parlementaire particulier, qui s'est déroulé le 19 octobre 2003, nous entendons montrer comment les références qui y ont été faites à la charia et à l'islam l'ont été - et le sont toujours - de manière contextuelle. À l'intérieur de l'Assemblée du Peuple, ce contexte est fait des contraintes qui s'exercent sur le traitement des questions qui y sont débattues et qui contribuent directement à leur configuration: (1) l'orientation vers des audiences, matérielles ou virtuelles; (2) l'alignement sur des considérations - des pertinences - d'ordre politique et législatif; (3) la référence aux exigences institutionnelles et procédurales. Tout au long du débat sur l'élévation de l'âge de garde des enfants, ces éléments de pertinence 
sont manifestés, explicités, utilisés par les membres de l'Assemblée à toutes fins législatives pratiques, c'est-à-dire dans l'accomplissement de leur activité de fabrique de la loi. Commençons avec l'extrait suivant.

\section{Muhammad AL-Habach ${ }^{1}$}

Monsieur le Président, chers collègues.

L'article 2 de la Constitution de la République arabe syrienne stipule que la religion du président de la République est l'islam. Le deuxième paragraphe de l'article 3 stipule que le droit (fiqh) islamique est une des sources principales de la législation.

En réalité, la question de la garde, comme le savent les spécialistes, fait partie des questions sur lesquelles la Loi (charia) ultime n'a pas voulu prononcer de propos définitif. Il n'y a pas de texte, ni dans le saint Coran, ni dans la noble Sunna, qui définisse l'âge de la garde. Dès lors, les vénérables jurisconsultes des différentes écoles se sont lancés dans la définition de l'âge de la garde, du choix des Chaféites pour [l'âge] de sept ans pour le garçon et dix ans pour la fille au choix des Malikites qui ont été jusqu'à dire que la garde du jeune homme [allait] jusqu'à sa majorité et pour la fille jusqu'à ce qu'elle se marie. Al-Hasan al-Basri a même été jusqu'à dire que la garde de la fille se prolongeait au-delà du mariage.

De toute manière, les options du droit islamique sont larges sur cette question et je pense que, quand nous ouvrons le corpus de droit islamique dans son ensemble et sans réserve, nous trouvons les options qui conviennent à toute époque et à tout lieu. Pour ce qui est du projet de loi qui est entre nos mains, j'ai participé à la discussion qui a eu lieu à la Commission des affaires constitutionnelles et législatives et j'ai été heureux du respect [manifesté] par tout le monde pour le droit islamique comme source de la législation et de l'attention [portée] aux options des imams - sur eux soit la satisfaction de Dieu. En fait, le [résultat] auquel la Commission est arrivée dans la définition de la durée de la garde à onze ans pour le garçon et à treize ans pour la fille représente un pas positif. Toutefois, s'agissant du paragraphe b de l'article lle juge a le droit d'étendre la durée de la garde à treize ans pour le garçon et à quinze ans pour la fille, s'il lui paraît que l'intérêt de l'enfant gardé l'impose), je propose de ne pas soumettre cette disposition parce qu'elle contraint la femme à en référer au juge pour la prolongation de sa garde des enfants, alors que l'homme est davantage en mesure d'en référer au juge.

Pour cela, je propose que la disposition soit comme suit: "La durée de la garde s'achève quand le garçon a atteint l'âge de treize ans et la fille l'âge de quinze ans ». Je pense que cet âge est celui qui correspond aux exigences et aux besoins qu'a la fille d'être sous la surveillance de sa mère, à cet âge précis, tandis que le garçon a besoin d'être à l'ombre de son père à treize ans pour

1. Religieux sunnite, député indépendant de la circonscription de Damas. 
plonger dans les tribulations de la vie, pour qu'il se prépare et s'entraîne à ses échéances.

Je pense qu'il est utile que la durée de la garde du garçon soit de treize ans et soit limitée pour la fille à quinze ans, et qu'en référer au juge revienne à l'homme qui est plus apte à cela, sachant que la loi a stipulé les échéances de la garde et qu'il est possible à l'homme de réduire la garde s'il existe des causes impératives. C'est pourquoi j'engage à ce que nous soyons à la recherche de la justice. La justice absolue, c'est quelque chose qui n'existe pas, sauf chez. Dieu Tout-Puissant. Nous nous efforçons de la chercher. De nombreuses mères ne peuvent pas veiller sur un enfant plus de deux ans et de nombreux pères ne sont pas en mesure de veiller sur un enfant.

\section{Le Président - interrompant}

Cher collègue Muhammad, votre proposition est de supprimer la mention (le juge a le droit d'étendre... etc.) et que [l'article] soit adopté sans elle. Est-ce que ce n'est pas le résumé du propos?

\section{Muhammad al-Habach-poursuivant}

Pour que ce sois clair, je demande que l'article soit adopté comme suit: "la durée de la garde s'achève quand le garçon a atteint l'âge de treize ans et la fille l'âge de quinze ans » et la suppression complète du paragraphe b. Je marque ma sympathie pour l'opinion de mon ami Monsieur 'Abd al-'Aziz al-Chami ${ }^{2}$ avec qui je suis d'accord quant à cette option. Merci.

L'intervention de Habach se présente comme une véritable argumentation fondée juridiquement. Elle inscrit en effet la légitimité du recours au répertoire islamique dans la Constitution elle-même. En ce sens, elle s'impose par l'effet d'une solidarité positive, au sens où l'entend Locke, c'est-à-dire d'une solidarité fondée sur la citoyenneté et la moralité (islamique dans notre cas) d'individus œuvrant à la réalisation de l'intérêt général. L'argumentaire de Habach suit le cours suivant: le droit islamique est constitutionnellement une source de la législation syrienne; la question de l'âge de la garde relève du droit islamique, mais celui-ci n'a pas établi de règle intangible en la matière; il est important de légiférer dans le respect de l'islam; toute élévation de l'âge de la garde va dans le bon sens; il faut toutefois tenir compte de l'accès inégal à la justice de l'homme et de la femme; mieux vaut donc éviter d'avoir à en recourir au juge et élever cet âge à sa limite " naturelle », qui varie entre filles et garçons; l'Assemblée à le devoir de tendre vers la justice, même si cela ne peut être absolu; il faut donc établir des principes, mais aussi faire en sorte qu'ils ne soient pas trop rigides. Habach tend à faire converger légalité et normalité, à donner à son évaluation normative de l'âge normal de la garde des enfants par la mère (quinze ans pour la fille, treize ans pour le garçon) la force d'une loi fondée sur la poursuite de l'intérêt général. C'est en cela précisément qu'il se distingue de l'intervention de Humsi (cf. infra),

2. Religieux sunnite, député indépendant de la circonscription d'Alep. 
qui prétend asseoir son argument sur la seule force de la transcendance. Habach, lui, vise à faire correspondre, dans une démarche jusnaturaliste par excellence, nature, société, loi morale et loi divine.

À la question du président qui l'interrompt, lui propose une synthèse de ce qu'il vient de dire et lui en demande confirmation, Habach donne une réponse technique et s'aligne sur la position d'un autre membre du Parlement (Chami). Ce dernier ne prend pas part au débat, ce qui laisse supposer qu'il est absent. Que Habach le salue au passage semble également indiquer qu'ils entretiennent des relations de complicité. En faisant mention de l'intervention de cet autre parlementaire, l'intervention de Habach montre comment le débat s'inscrit dans une séquence longue où prennent place d'autres interventions relatives au même objet (qui forment, toutes ensemble, un réseau dialogique). C'est illustratif d'une des multiples façons dont les discours trouvent à se configurer intertextuellement. L'incorporation de la voix d'autrui (Chami), d'une tradition textuelle (al-Hasan al-Basri), de références dogmatiques abstraites (les Chaféites et les Malikites) et de la Constitution permettent à Habach de proposer un discours argumenté dont la validité procède du cumul de ces autorités et de leur agencement.

Tournons-nous à présent vers l'intervention d'une députée.

\section{Mme AL-Humsi ${ }^{3}$}

\section{Au nom de Dieu le Clément le Miséricordieux}

"Notre Seigneur, donne-nous, en nos épouses et nos descendants la joie des yeux et fais de nous un guide pour les pieux »(Coran, 25:74). Vérité du Dieu Majestueux.

La Loi islamique indulgente confirme que la fermme est un être humain doué de dignité et d'une personnalité juridique indépendante. Elle a pratiqué, depuis l'émergence de l'islam, c'est-à-dire depuis plus de mille quatre cents ans, la politique, l'allégeance et le combat sur la voie de Dieu. Elle n'a jamais été éloignée des affaires de la société et de la vie publique.

Et Dieu - gloire à Lui le Très-Haut! - quand Il a créé l'être et a créé un mode de vie et de subsistance complémentaire, a regardé les gens avec équanimité, qu'ils soient hommes ou fermmes, et Il n'a pas distingué entre eux. Tous deux se complètent dans cette vie et chacun a sa place.

Je demande protection à Dieu contre Satan le Lapidé, Au nom de Dieu le Clément le Miséricordieux

"Et celui qui fait de bonnes ouvres, homme ou femme, tout en étant croyant, ceux-là rentreront au Paradis et ne subiront pas l'injustice, fût-ce d'un creux de noyau »(Coran 4:124). Vérité du Dieu Majestueux.

3. Sunnite, députée FNP de la circonscription de Damas. 
L'Envoyé de Dieu - que Dieu le bénisse et lui donne la paix-a particulièrement mentionnéle dévouement de la mère, si bien qu'il a ordonné à celui qui lui posait la question: "Ô Envoyé de Dieu, quelle est la personne la plus digne de mon compagnonnage vertueux? - Ta mère » dit-il. "Qui ensuite? - Ta mère - Qui ensuite? - Ta mère - Qui ensuite? - Ton père » répondit-il.

Et parmi les propos de notre seigneur Jésus - sur lui soit la paix - dans la sourate de Marie:

Je demande protection à Dieu contre Satan le Lapidé, Au nom de Dieu le Clément le Miséricordieux

"Et la bonté envers ma mère, et Il ne m'a fait ni violent ni malheureux » (Coran 19:32).

Il est rapporté par un des compagnons que chaque fois qu'il s'en allait de chez sa mère, il la saluait et disait: "la paix soit sur toi ô mère, et la clémence de Dieu et Ses bénédictions, Dieu te fut Clément de la façon dont tu m'as élevé [quand j'étais] petit; et elle lui répondait en disant: sur toi soit la paix et la clémence de Dieu et Ses bénédictions: Dieu te fut Clément de la façon dont tu m'as aimée filialement [quand tu étais] grand» (al-Adab al-mufrad de Bukhari).

Dans les Lois célestes, est révélé ce qui est pour le bien de l'être humain et pour la réalisation de la justice dans la société. Elles sont fondées sur ce qu'il y a de bon dans les êtres humains. Les êtres humains sont toutefois faillibles et vulnérables, c'est pour cela qu'il faut nécessairement que soit promulgué un texte organisateur qui règle les relations des gens entre eux, et cela aussi à l'intérieur d'une seule et même famille.

Le projet de loi d'élévation de l'âge de la garde n'est pas une demande féminine. C'est une aspiration de la femme aussi bien que de l'homme. Les femmes sont les compagnes des hommes. L'homme, c'est le fils, le frère, l'époux et le père. Nous autres, par la grâce de Dieu, nous jouissons dans cette région de liens familiaux importants qui font de nous une société cohésive. Il est de notoriété dans notre pays que le père a de la peine lors du mariage de sa fille, de peur qu'elle ne s'en aille vers une vie qui ne puisse s'apparenter à celle que son père lui a offerte. C'est comment, alors qu'il la voit dans certains cas, elle qui s'est habituée à lui, souffrir de la séparation, qu'il [en vient à] la garder [avec lui].

L'élévation de l'âge de la garde est à quinze ans pour la fille et à treize ans pour le fils, après quoi ils ont atteint un degré de développement psychique prescrivant de leur laisser le libre choix de vivre chez leur mère ou chez leur père. C'est ce qu'a indiqué l'Envoyé - que Dieu prie sur lui et lui donne la paix - quand est venue à lui une femme et qu'elle a dit: "Ô Envoyé de Dieu! Mon époux veut partir avec mon fils alors qu'un puits [qui] arrose ses raisins m'est profitable. "L'Envoyé a dit au fils: c'est ton père et c'est ta mère, prends la main de celui des deux que tu veux. Il a alors pris la main de sa mère et elle s'est éloignée avec lui ». Merci. 


\section{Le Président}

Je suis extrêmement patient et je poursuivrai avec vous jusqu'à sept heure du matin. Vous disposez du temps et vous pouvez évaluer le temps. Les limites naturelles pour tout intervenant sont de quatre minutes au maximum et si chacun d'entre vous parle les quatre minutes qui lui sont allouées, nous poursuivrons jusqu'à minuit. J'espère à nouveau que nous restreindrons le sujet radicalement aux deux années auxquelles l'âge de la garde a été élevé. Sont-elles acceptables ou non? La parole est à [notre] collègue Muhammad al-Habach. [...]

Le discours de Huda al-Humsi se situe résolument dans le registre de l'islam et de la charia. Il se présente sous la forme du syllogisme suivant: (a) l'intérêt de l'enfant est d'être gardé en fonction de ses besoins spécifiques; (b) dans une société islamique, c'est la mère qui assure au mieux la protection des intérêts de l'enfant; (c) le droit syrien, en tant que droit respectueux des valeurs islamiques de la société, veille à l'intérêt de l'enfant en prolongeant la durée de sa garde par la mère. Apparaît en filigrane l'objectif pratique (et évident dans un cadre parlementaire) d'appuyer le projet d'élévation de l'âge de la garde par la mère et ainsi d'amener une majorité de parlementaires à voter en ce sens. Dans le débat qui nous occupe, la question de la pertinence du registre islamique n'est jamais posée. Elle est, au contraire, constamment avancée, sur le mode de l'évidence, comme dans l'intervention de Humsi qui voit se succéder des références religieuses donnant au discours la force d'un argument d'autorité (d'autant qu'il n'est personne pour le contester, que ce soit pour des raisons de compétence dans les questions religieuses ou pour des raisons de légitimité confessionnelle), ou sur celui de l'argumentation, comme dans l'intervention de Muhammad al-Habach.

Mais, au-delà du contenu, ce qui est marquant dans cet extrait, c'est la capacité du président de l'Assemblée à contrôler et orienter le débat. Quel que soit le sujet et en dépit de son caractère sacré, on observe qu'il organise le débat, y prend une part active et le dirige dans le sens de ses préférences et, entre autres choses, d'une gestion serrée du temps de parole.

Si l'on se tourne à présent vers la question de la pertinence politique et législative, on peut observer comment des répertoires argumentatifs et des stratégies discursives s'entremêlent étroitement.

\section{Mme WA'D KHADdAM $^{4}$}

\section{Monsieur le Président, chers collègues.}

La garde est un droit de l'enfant et elle a été légiférée dans son intérêt. Tel est le principe fondamental qu'ont établi le fiqh et la loi. L'interprétation se fait dans l'intérêt de [l'enfant] gardé dans tous les domaines. Du fait que la question de

4. Sunnite, députée FNP de la circonscription de Tartous. 
l'éducation des enfants diffère dans les sociétés modernes de ce qu'elle [était] dans les sociétés anciennes et impose le changement de l'infrastructure de ces sociétés, on demande d'allonger la durée de la garde.

Tout comme l'application de la loi surl'enseignement fondamental obligatoire jusqu'à la fin du cycle préparatoire, on demande l'allongement de la durée de la garde en sorte que l'[enfant] gardé n'ait pas à changer le lieu de résidence et de vie auquel il est habitué et, en particulier, l'école et l'environnement scolaire.

À la base, les opinions des jurisconsultes et des législateurs ont différé sur la durée de la garde en fonction des différences de situation sociale et des différences dans les besoins éducatifs des enfants.

C'est pour cela que nous considérons que l'amendement proposé protège cet intérêt et je demande que soit [donnée] au juge l'appréciation du meilleur intérêt de l'[enfant] gardé dans chaque affaire.

Avec l'augmentation de la pension alimentaire destinée à chaque enfant et la garantie du logement de l'[enfant] gardé, ce qui est une question importante qu'on ne peut pas supprimer ou absorber dans son règlement financier, dans la mesure où la question du logement est devenue difficile et exorbitante et où la gardienne ne peut plus la garantir dans la plupart des cas, en particulier si la mère a plus d'un enfant, si la maison de sa famille est petite et si elle ne travaille pas. Cette question a été résolue en Tunisie, Algérie, Égypte, Irak, Yémen, Koweït.

Pour tout ceci, je suis en faveur du projet de loi et d'une promulgation rapide. Merci.

Quand elle prend la parole, Wa'd Khaddam apparaît explicitement comme une femme. Cela se manifeste aussi bien physiquement que par l'indexation générique opérée par la mention du prénom (Wa'd) par le président de l'Assemblée, quand il lui donne la parole, et se traduit dans la retranscription officielle par l'utilisation de la civilité devant le nom («Mme »). Cette catégorisation pave la voie, logiquement bien que pas nécessairement, du recours à un dispositif catégoriel organisé autour de l'enfant. Celui-ci est présenté comme doté d'intérêts et de droits (ses besoins en matière d'éducation, d'enseignement, de moyens de subsistance, de garde) qu'il convient de préserver. C'est au droit, à la fois musulman et moderne, qu'il revient d'œuvrer à cette fin. Le raisonnement de l'orateur procède donc du syllogisme suivant: (a) l'intérêt de l'enfant est d'être gardé en fonction de ses besoins spécifiques; (b) dans une société moderne, l'enfant a besoin d'être gardé par sa mère plus longtemps; (c) le droit syrien veille à l'intérêt de l'enfant dans la société moderne en prolongeant la durée de sa garde par la mère. Le pivot de ce syllogisme réside dans la notion de modernité. Le raisonnement procède ainsi sur la base d'un implicite en fonction duquel l'éducation dans une société moderne imposerait de confier l'enfant plus longtemps à sa mère. Autrement dit, le dispositif catégoriel que nous voyons 
à l'œuvre est celui qui unit l'enfant à sa mère pour les besoins d'une éducation dont la durée augmente avec la modernité de la société.

C'est exactement le même raisonnement qui est suivi par le député Ghalib 'Aniz ${ }^{5}$, à ceci près - mais la différence est fondamentale - que c'est le père qui est ici présenté comme le plus apte à préparer l'enfant à la confrontation aux difficultés de la vie moderne.

\section{GHALIB `ANIZ}

Monsieur le Président, Mesdames et Messieurs, chers collègues.

Dieu a gratifié la femme de qualités et de mérites énormes et Il l'a élevée à une place éminente dans la société. La réussite et le succès n'arrivent qu'avec l'accord des deux parents, la mère et le père.

La femme constitue la moitié de la société et l'homme l'autre moitié. On dit dans le proverbe populaire: l'homme achète des grains, la femme en fait du pain. Qui pourrait priver la femme de son droit? Elle est l'artisane des hommes, elle est la mère, la sour et l'épouse et, sans elle, ils ne prospèrent pas. Le Prophète - que Dieu le bénisse et lui donne la paix - dit: "les femmes sont les compagnes des hommes ». La femme, c'est celle qui est tendre et affectueuse, elle est la base et le fondement du succès des familles, de leur développement et de leur bien-être.

Le bonheur de la femme se reflète positivement sur le bonheur de la famille.

Mais cette affection ardente et cette tendresse énorme la rendent incapable - je ne dis pas toujours mais le plus souvent - de contrôler les jeunes garçons quand ils deviennent adolescents, de même que les filles quand elles atteignent un âge plus avancé.

La garde, cela ressort de son nom même, concerne un âge précoce et non un âge plus avancé. Il y a des femmes qui portent le bien en elle, tout comme des hommes, et l'inverse aussi.

Peut-être que l'âge auquel ce projet est arrivé, après une étude extensive, est le plus adéquat et le plus proche de la raison, de chaque opinion, de sa direction et de son but. Je suis pour l'amendement de la Commission, mais [je souhaite] que cette extension soit telle qu'il n'y ait pas lieu au différend judiciaire et à l'antagonisme, que cette extension que la Commission a proposée se fasse à la demande et dans la chambre de délibération, en sorte qu'aucun des deux époux ne soit contraint à présenter une requête et à la poursuivre en longueur, que s'exprime l'opinion d'un comité de deux arbitres, comme c'est le cas en matière de séparation (un arbitre de sa famille à lui et un arbitre de sa famille à elle) et d'une personne distincte que les deux arbitres choisissent, connu

5. Avocat sunnite, député indépendant de la circonscription de Damas. 
sous le nom de comité d'arbitres, un arbitre de sa famille à lui, un arbitre de sa famille à elle et une personne distincte que choisissent les deux arbitres. Peut-être le bien viendra-t-il de cela, Dieu est le Conciliateur. L'instruction n'a pas de rapport avec ce sujet. Merci.

Nous voyons dès lors comment le débat parlementaire fonctionne sur le mode de la solidarité sans consensus: solidarité sur les termes fondamentaux (modernité, droit, intérêt de l'enfant); dissensus sur les conséquences à en tirer (droits de la mère contre droits du père). C'est, en quelque sorte, à une compétition sur la maîtrise de la définition des éléments propres à un dispositif catégoriel que nous assistons. Le dispositif en question est celui de la famille, avec sa collection d'éléments (les parents, les enfants), dont certains sont appariés (père-mère, enfants-parents, sœurs-frères). Des activités sont liées aux catégories de ce dispositif: se marier, divorcer, vivre ensemble, éduquer, garder, entretenir, etc. Ce sont les relations entre les différents éléments appariés et les activités qui sont liées au dispositif et à ses éléments qui constituent l'enjeu de ce conflit, non pas de catégorisation, mais d' « apportionnement » des droits et devoirs liés aux différents éléments du dispositif. L'on voit ici comment les catégories de sens commun trouvent à se déployer de manière clivée, sur la base des mêmes composantes et des mêmes jeux de rattachement, en prétendant toujours à la normalitél naturalité des configurations relationnelles qu'elles établissent. L'on peut de la sorte observer l'existence de deux types symétriques de conflits: d'une part, les conflits de catégorisation (par exemple, la qualification de " papa », de " père » ou de " chef de famille »); de l'autre, les conflits d'apportionnement, où ce n'est plus l'appartenance à une catégorie qui pose problème mais la détermination des droits et devoirs normaux/naturels qui s'y rattachent (le père est " responsable », la mère est " tendre », etc.).

Cette étude rapide de quelques tours de parole à l'occasion de la discussion d'un projet de loi nous a permis de voir à quel point les débats parlementaires sont contraints par la recherche d'une pertinence politique et législative, dont la production se déploie publiquement dans des jeux de langage dont les députés sont familiers, en fonction de ce qui, pour eux, peut être significatif. La façon de parler de sujets tels que l'islam et la charia s'avère donc éminemment dépendante du contexte dans lequel ils sont thématisés. Dès lors que c'est à l'intérieur du Parlement syrien, des contraintes institutionnelles et procédurales viennent s'ajouter à des contraintes argumentatives et sémantiques pour donner à ces sujets une forme tout à fait singulière, dont il est de ce fait impossible de rendre compte en généralité. Il n'y a pas un islam syrien, ni même des islams syriens, mais autant d'islams qu'il n'y a de lieux et de gens pour en parler et les pratiquer, d'une manière modelée irrémédiablement par le contexte et les finalités pratiques propres à l'activité de tout un chacun. 